\title{
Time-series Doppler imaging of the red giant HD 208472
}

\section{Active longitudes and differential rotation $\star, \star \star$}

\author{
O. Özdarcan ${ }^{1,2}$, T. A. Carroll ${ }^{1}$, A. Künstler ${ }^{1}$, K. G. Strassmeier ${ }^{1}$, S. Evren ${ }^{2}$, M. Weber ${ }^{1}$, and T. Granzer ${ }^{1}$ \\ ${ }^{1}$ Leibniz Institute for Astrophysics Potsdam (AIP), An der Sternwarte 16, 14482 Potsdam, Germany \\ 2 Ege University, Faculty of Science, Astronomy and Space Sciences Department, 35100 Bornova, İzmir, Turkey \\ e-mail: orkun.ozdarcan@ege.edu.tr
}

Received 18 March 2016 / Accepted 7 July 2016

\begin{abstract}
Context. HD 208472 is among the most active RS CVn binaries with cool starspots. Decade-long photometry has shown that the spots seem to change their longitudinal appearance with a period of about six years, coherent with brightness variations.

Aims. Our aim is to spatially resolve the stellar surface of HD 208472 and relate the photometric results to the true longitudinal and latitudinal spot appearance. Furthermore, we investigate the surface differential rotation pattern of the star.

Methods. We employed three years of high-resolution spectroscopic data with a high signal-to-noise ratio (S/N) from the STELLA robotic observatory and determined new and more precise stellar physical parameters. Precalculated synthetic spectra were fit to each of these spectra, and we provide new spot-corrected orbital elements. A sample of 34 absorption lines per spectrum was used to calculate mean line profiles with a $\mathrm{S} / \mathrm{N}$ of several hundred. A total of 13 temperature Doppler images were reconstructed from these line profiles with the inversion code iMap. Differential rotation was investigated by cross-correlating successive Doppler images in each observing season.

Results. Spots on HD 208472 are distributed preferably at high latitudes and less frequently around mid-to-low latitudes. No polarcap like structure is seen at any epoch. We observed a flip-flop event between 2009 and 2010, manifested as a flip of the spot activity from phase 0.0 to phase 0.5 , while the overall brightness of the star continued to increase and reached an all-time maximum in 2014 . Cross-correlation of successive Doppler images suggests a solar-like differential rotation that is $\approx 15$ times weaker than that of the Sun.
\end{abstract}

Key words. stars: activity - stars: imaging - starspots - stars: individual: HD 208472

\section{Introduction}

Well-sampled photometric monitoring of spotted cool stars allows tracing photospheric brightness variations that are caused by rotational modulation. Long-term photometric data moreover allow investigating spot activity cycles and relate inherent changes of the surface spot distribution to photometric period variations, both of which are an indicator of the underlying surface differential rotation. Phenomena such as active longitudes (Henry at al. 1995; Jetsu 1996) and flip-flops of active longitudes (Jetsu et al. 1991; Berdyugina \& Tuominen 1998) were revealed by such long-term photometry, but they are far from being fully understood. Holzwarth \& Schüssler (2003a,b) demonstrated on theoretical grounds that the tidal effects of a companion star in a binary system may alter the surface distribution of spots by breaking the axial symmetry, which could lead to preferred longitudes. Magnetic dynamo simulations (Fluri \& Berdyugina 2004; Moss 2005; Elstner \& Korhonen 2005) of the flip-flop phenomenon basically concluded that the axisymmetric and the non-axisymmetric (magnetic) field components must coexist to

\footnotetext{
$\star$ Based on data obtained with the STELLA robotic telescope in Tenerife, an AIP facility jointly operated by AIP and IAC, and the Potsdam Automatic Photoelectric Telescopes (APT) in Arizona, jointly operated by AIP and Fairborn Observatory.

$\star \star$ Radial velocity measurements are only available at the CDS via anonymous ftp to cdsarc.u-strasbg. fr (130.79.128.5) or via http://cdsarc.u-strasbg.fr/viz-bin/qcat?J/A+A/593/A123
}

produce a flip-flop in the first place. These studies highlighted that meridional circulation and differential rotation are a prerequisite for this type of dynamo but do not yet allow predicting the relation between differential rotation and flip-flop period, for example. Recently, Roettenbacher et al. (2015a,b) showed that there might be ambiguity in interpreting light curves of RS CVn binaries in terms of persistent active longitudes and ellipsoidal distortion. In this case, some part of the light curve might be caused by binarity effects and not by cool spots.

For the characterization of differential surface rotation, timeseries Doppler imaging became the most powerful method (see, e.g., Donati \& Collier Cameron 1997; Strassmeier 2009; Künstler et al. 2015; Kővári et al. 2016). The differential rotation signature is either based on the longitudinal crosscorrelation of images from successive rotation cycles or on the addition of the image shear as an additional free parameter in the line-profile inversion itself. Neither of these variants is free of problems, see Dunstone et al. (2008) for a comparison, but both were successfully applied to a few single and binary stars in different evolutionary stages (e.g., Donati et al. 2003; Dunstone et al. 2008; Kővári et al. 2012). For main-sequence stars, only solar-type differential rotation (equator rotates faster than poles) was found so far, while anti-solar differential rotation (poles rotate faster than equator) was found for a few fast-rotating giants (Weber et al. 2005). Kitchatinov \& Rüdiger (2004) have shown that fast meridional circulation in a very deep 
convection zone with magnetically induced thermal inhomogeneities would lead to anti-solar differential rotation. However, at least in some cases, different authors found contradictory results for one and the same target, for example, for the K sub-giant of the RS CVn binary HR 1099, for which Vogt et al. (1999) analyzed high-resolution unpolarized spectroscopic data and found weak anti-solar differential rotation, while Petit et al. (2004) used high-resolution polarized spectra and found solar-type differential rotation. It is mostly assumed that the spots follow the rotation of the surface, while differential rotation is investigated by photometric spot modeling and/or time-series Doppler imaging. However, Korhonen \& Elstner (2011) suggested that large spot structures could reflect geometric properties of the largescale dynamo and not the surface rotation. Still, the spots are the tracers we rely on.

HD 208472 (V2075 Cyg, HIP 108198, $V=7$ m.4, $P_{\text {phot }}=$ $22.4 \mathrm{~d}$ ) is a conspicuous target that seems to show active spot longitudes and an activity cycle with a period of about six years Özdarcan et al. (2010; hereafter Paper I). In 1991, W. Bidelman discovered its strong $\mathrm{CaII} \mathrm{H}$ and $\mathrm{K}$ emission, and a number of follow-up studies revealed and confirmed that the system is a single-lined spectroscopic binary with a G8III primary component on a nearly circular orbit that exhibits rotationally modulated light variations with variable light-curve amplitude (Henry at al. 1995; Fekel et al. 1999; Strassmeier et al. 1999; Koen \& Eyer 2002). It was also suggested that the system may be a good candidate for Doppler imaging (Henry at al. 1995). First such attempts for Doppler imaging were made by Weber et al. (2001), Weber (2004) and Weber et al. (2005), whose initial study was based on 70 consecutive nights of spectroscopic observations with the NSO McMath-Pierce telescope between 1996 and 1997. They found that spots at that time were concentrated at low and intermediate latitudes without any structures close to the one visible pole of the star. They applied the sheared-image method and found a weak anti-solar differential rotation of $\alpha=-0.04 \pm 0.02$, which, however, was not considered a conclusive result. Erdem et al. (2009) estimated an $\alpha$ parameter of the same amount, but relative to the orbital period, through photometric spot modeling based on $B V R_{\mathrm{c}} I_{\mathrm{c}}$ photometry in 2006. More comprehensive photometry was presented in our Paper I, where we analyzed 17 yrs of data from Automatic Photoelectric Telescopes (APT). A spot-modeling and photometric-period analysis led us to a differential rotation coefficient of $\alpha=0.004 \pm 0.010$, which practically indicated a nondetection of differential rotation. Furthermore, we found that the longitudinal position of spots varied coherently with the mean brightness and seemed to exhibit a $6.28 \mathrm{yr}$ period. This cyclic variation was interpreted as a stellar analog of the solar 11-yr sunspot cycle.

In the current study, we present new high-resolution timeseries spectroscopy of the system from 2009-2011 together with contemporaneous $V$-band photometry. We redetermine precise atmospheric parameters, obtain an improved spectroscopic orbit ,and investigate the evolutionary status of the primary star. The unique time-series data from STELLA enables us to obtain Doppler images of the star for many successive rotational cycles, and for three consecutive years. We first summarize the instrumental setup and the data collection and reduction procedures in Sect. 2. In Sect. 3 we derive stellar atmospheric parameters, spectroscopic orbital elements and physical properties of the system. Section 4 comprises the Doppler imaging of the primary star, including data preparation and a brief description of the $i$ Map inversion code. We present a total of 13 surface maps and their analysis in Sect. 5. In the final section, we summarize and discuss our results.

\section{Observations and data reductions}

Spectroscopic observations were carried out with the robotic STELLA facility on Tenerife, Spain, from March 22, 2009 to July 20, 2011. All spectra were obtained with the fiber-fed STELLA Echelle Spectrograph (SES) with a resolving power of $R=55000$ corresponding to a two-pixel resolution of $0.12 \AA$ at $6400 \AA$. The spectra cover the wavelength range $3900-8800 \AA$. In most cases, a single exposure was taken per available night. On some occasions, two successive spectra were obtained. Exposure time was set to $7200 \mathrm{~s}$ during the 2009 observing season, and $4500 \mathrm{~s}$ thereafter. The average signal-to-noise ratio $(\mathrm{S} / \mathrm{N})$ varied between 240 and 160 for the three observing seasons. By 2010, the efficiency for coupling the starlight into the fiber was significantly improved, and equally good $\mathrm{S} / \mathrm{N}$ spectra were obtained with a shorter exposure time. In total, 229 usable spectra were acquired during three years.

Raw spectra were reduced with the SES data reduction pipeline, which is mainly based on $\mathrm{IRAF}^{1}$ command-language scripts (Weber et al. 2008). CCD images are corrected for bad pixel and cosmic-ray impacts. Average overscan is calculated and subtracted from each image. An already overscan-subtracted master bias frame is applied to the images to remove bias levels. Nightly obtained flat-field images (around dusk, dawn, and midnight) are used to produce a master flat-field image of the related night, and this master image is normalized to unity. Then target spectra are divided by this master flat. Scattered-light correction is done from the inter-order space, and one-dimensional spectra are extracted with the standard IRAF optimal-extraction routine. After this, a wavelength calibration is applied by using consecutively recorded Th-Ar spectra. Finally, continuum normalization of the extracted spectral orders is done by dividing with a fluxnormalized synthetic spectrum of spectral classification just like HD 208472.

Photometric observations were conducted with the T7 0.75 m APT Amadeus at Fairborn Observatory in southern Arizona (Strassmeier et al. 1997). Its data were extensively used and described in Paper I, and we refer to this paper for instrumental and observational details.

\section{Astrophysical parameters}

\subsection{Unspotted brightness}

Figure 1a shows all available Johnson $V$-band data of HD 208472. With the addition of our new APT data for the time range from 2010 to 2015, the photometric coverage now extends continuously over 23 yrs. We determined the peak-to-peak light curve amplitudes and minimum brightness levels for each observing season and found that the system was never brighter than by the end of 2014 , reaching a $V$ magnitude of $7 \mathrm{~m} .13 \pm 0.01$. This is even brighter than the predicted unspotted (apparent) brightness of $7 \mathrm{~m} .323$ previously estimated in Paper I. Using the minimum brightness vs. amplitude method of Oláh et al. (1997), we find a new value for the unspotted $V$ brightness of 7 m04 (Fig. 1b). Using the interstellar reddening obtained in Paper I

\footnotetext{
1 The Image Reduction and Analysis Facility is hosted by the National Optical Astronomy Observatories in Tucson, Arizona at URL iraf.noao. edu.
} 

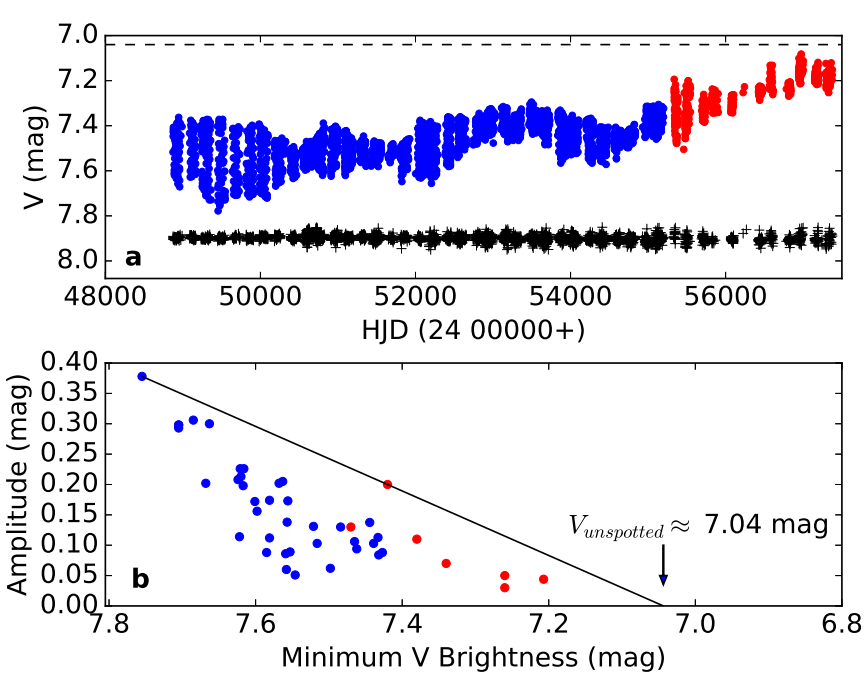

Fig. 1. a) Long-term $V$-band photometry of HD 208472. The dashed line denotes the updated unspotted brightness level. The blue filled dots show data from Paper I, while the red dots denote the recent data. Plus signs show differential magnitudes in the sense of check-minuscomparison star. These measurements are shifted by a constant amount and plotted on a common scale with measurements of the variable star. b) Minimum brightness vs. $V$ amplitude diagram. The straight line is a linear representation of the upper envelope of the scatter.

$(E(B-V)=0 \mathrm{~m} \cdot 127)$, we revise the intrinsic unspotted $V$ brightness of HD 208472 to $6.65 \pm 0.01$.

This updated value together with the HIPPARCos distance of $153 \mathrm{pc}$ (van Leeuwen 2007) modifies the absolute $V$ magnitude of HD 208472 to $+0^{\mathrm{m}} .73 \pm 0.18$. Its luminosity is therefore $64 \pm$ $5 L_{\odot}$ (adopting $M_{\mathrm{bol}, \odot}=+4$ m.74).

\subsection{Orbital elements}

Radial velocities (RV) from STELLA-SES spectra were derived from an order-by-order cross correlation with a synthetic template spectrum and then averaged. A total of 60 orders out of the 80 available were used, and a synthetic template spectrum of a G8 giant was adopted. The standard (external) error of a single STELLA-SES observation of HD 208472 is $\approx 30 \mathrm{~m} \mathrm{~s}^{-1}$. No systemic zero-point shift was added to the data in this paper, but see Strassmeier et al. (2012) for its determination with respect to the CORAVEL reference system. The complete data set of $229 \mathrm{RVs}$ is available in electronic form at the CDS Strasbourg.

For an initial orbital solution we adopted the elements from Fekel et al. (1999) as starting values. The preliminary solution showed clear and systematic residuals with a full amplitude of up to $1 \mathrm{~km} \mathrm{~s}^{-1}$ (Fig. 2a). Two anti-phase waves can be identified (lower panel of the figure), indicating that two spots or spot groups traversed the stellar disk during the time of observation. We applied a discrete Fourier transform to the residuals using the Period04 (Lenz \& Breger 2004) software package and searched for significant periods. This was done separately for the individual observing season. In 2010, we subdivided the time coverage into two data groups because the variation pattern had changed between the first and second half of the season. The resulting periods with significance of $5 \sigma$ or more in amplitude are tabulated in Table 1 together with their amplitudes. The dominant period is close to the photometric (=rotational) period in 2010 and 2011, while the other periods are just the first, second, and third harmonics of it. In 2009, half of the photometric period is the most dominant one and the other periods are the photometric period
Table 1. Periodogram results of radial velocity residuals.

\begin{tabular}{crc}
\hline \hline Year & Period $(\mathrm{d})$ & Amplitude $\left(\mathrm{km} \mathrm{s}^{-1}\right)$ \\
\hline 2009 & $11.127 \pm 0.006$ & $0.48 \pm 0.01$ \\
& $22.882 \pm 0.052$ & $0.24 \pm 0.01$ \\
& $7.382 \pm 0.018$ & $0.07 \pm 0.01$ \\
& $25.322 \pm 0.189$ & $0.07 \pm 0.01$ \\
\hline $2010-\mathrm{A}$ & $22.642 \pm 0.111$ & $0.39 \pm 0.02$ \\
& $11.368 \pm 0.034$ & $0.32 \pm 0.02$ \\
& $7.375 \pm 0.020$ & $0.23 \pm 0.02$ \\
& $5.580 \pm 0.030$ & $0.09 \pm 0.02$ \\
\hline $2010-\mathrm{B}$ & $22.215 \pm 0.170$ & $0.33 \pm 0.02$ \\
& $7.484 \pm 0.034$ & $0.19 \pm 0.02$ \\
& $10.857 \pm 0.081$ & $0.17 \pm 0.02$ \\
\hline 2011 & $23.059 \pm 0.375$ & $0.28 \pm 0.03$ \\
\hline
\end{tabular}

Table 2. Spectroscopic orbital elements.

\begin{tabular}{lcc}
\hline \hline & Uncorrected & Spot corrected \\
\hline$P_{\text {orb }}($ days $)$ & $22.61942 \pm 0.00066$ & $22.61940 \pm 0.00015$ \\
$T_{0}(\mathrm{HJD} 2449+)$ & $252.414 \pm 0.18$ & $252.419 \pm 0.041$ \\
$\gamma\left(\mathrm{km} \mathrm{s}^{-1}\right)$ & $10.968 \pm 0.012$ & $10.974 \pm 0.003$ \\
$K_{1}\left(\mathrm{~km} \mathrm{~s}^{-1}\right)$ & $16.355 \pm 0.035$ & $16.345 \pm 0.008$ \\
$e($ adopted $)$ & 0 & 0 \\
$\omega$ & $\ldots$ & $\ldots$ \\
$a_{1} \sin i\left(10^{6} \mathrm{~km}\right)$ & $5.087 \pm 0.011$ & $5.084 \pm 0.003$ \\
$f(m)\left(M_{\odot}\right)$ & $0.01025 \pm 0.00006$ & $0.01023 \pm 0.00001$ \\
fit rms $\left(\mathrm{m} \mathrm{s}^{-1}\right)$ & 380 & 88 \\
\hline
\end{tabular}

Notes. The elements are for the unaltered RV data (Col. "Uncorrected") and for starspot-jitter removed data (Col. "Spot corrected").

itself and its other harmonics. This confirms that the RV scatter is due to starspots migrating in and out of sight and not due to an as yet undiscovered planet.

In the next step, we pre-whitened the RV measurements of every individual observing sequence with a least-squares fit from the periods in Table1. These data are called the spot-corrected velocities. We then repeated the orbital solution and obtained the final elements given in Table 2. Figure $2 b$ shows the best orbital fit with respect to the spot-corrected RVs and its residuals. We note that the orbital elements remain almost identical, but the errors were reduced on average by a factor of 4 .

\subsection{Astrophysical parameters}

Effective temperature, surface gravity, metallicity and microturbulence were determined from each individual SES spectrum with the PARSES pipeline (Allende Prieto 2004; Allende Prieto et al. 2006; Jovanovic et al. 2013). This fits precalculated grids of synthetic spectra to selected wavelength regions of the observed spectra. Grids of synthetic spectra were calculated from MARCS model atmospheres in local thermodynamic equilibrium (LTE) (Gustafsson et al. 2008). The pipeline searches for the best-fit synthetic spectrum by minimizing the difference between the observed and computed spectrum and then adopts its parameters as its best solution. In addition to the above four atmospheric parameters, a line-broadening parameter consisting of the combined effect of $v \sin i$ and macroturbulence 

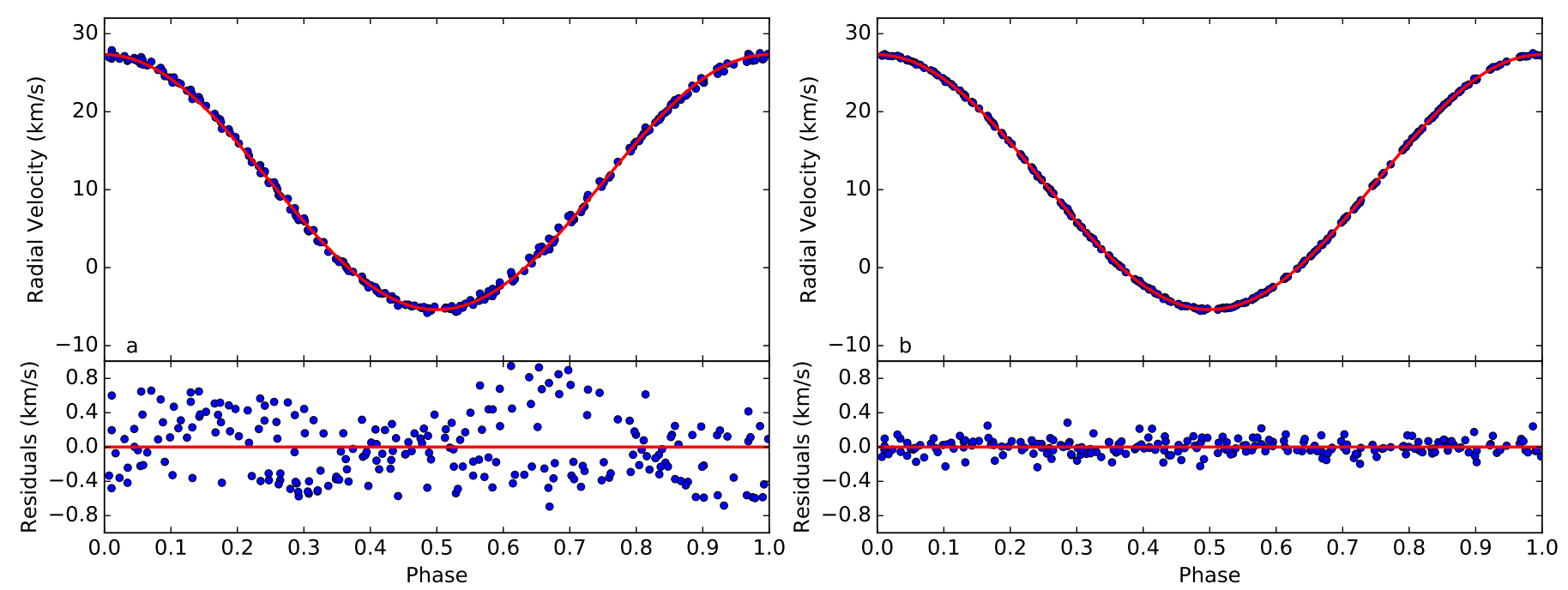

Fig. 2. a) Radial velocities (dots) before spot correction and the representation of the orbital fit (line). The bottom panel shows the residuals. b) Spot-corrected radial velocities and the final fit for the orbital elements. The rms of the fit decreased by a factor four with respect to the uncorrected velocities.
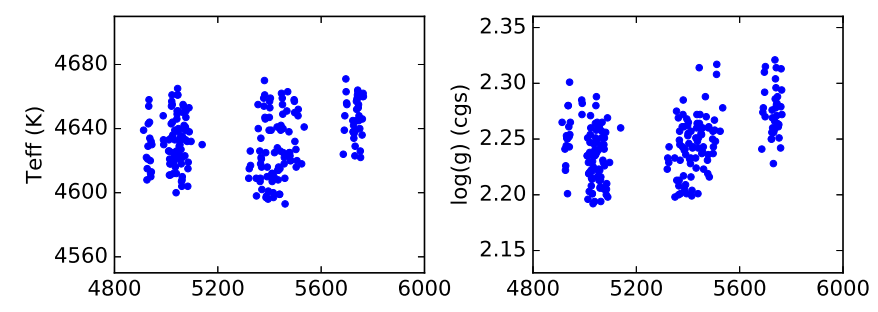

Table 3. Astrophysical parameters of HD 208472. Errors quoted are internal errors.

\begin{tabular}{ll}
\hline \hline Parameter & Value \\
\hline Spectral Type & K1 III \\
$V_{0}$ & $6 \cdot 65$ \\
Bolometric correction & $-0^{\mathrm{m}} \cdot 51$ \\
$M_{V}$ & $0^{\mathrm{m}} \cdot 73 \pm 0.18$ \\
$M_{\text {bol }}$ & 0.22 \\
$v$ sin $i$ & $19.4 \pm 0.2 \mathrm{~km} \mathrm{~s}^{-1}$ \\
Microturbulence & $1.71 \pm 0.05 \mathrm{~km} \mathrm{~s}^{-1}$ \\
Macroturbulence & $5 \mathrm{~km} \mathrm{~s}$ (adopted $^{-1}$ \\
[Fe/H] (solar) & $-0.49 \pm 0.01$ \\
$T_{\text {eff }}$ & $4630 \pm 18 \mathrm{~K}$ \\
Luminosity & $64 \pm 5 L_{\odot}$ \\
log $g$ (cgs) & $2.25 \pm 0.03$ \\
Radius & $12.5 \pm 0.5 R_{\odot}$ \\
Inclination & $44^{\circ} \pm 10^{\circ}$ \\
Mass & $1.2 \pm 0.1 M_{\odot}$ \\
Age & $\sim 3.5 \mathrm{Gyr}$ \\
\hline
\end{tabular}

was adopted as a free parameter during the fitting process. According to the atmospheric properties of the star, an assumed macroturbulence of $5 \mathrm{~km} \mathrm{~s}^{-1}$ was adopted from Gray (2005) and kept fixed.

The PARSES results for 218 SES spectra are plotted as a function of Julian date in Fig. 3. An effective temperature of $4,630 \mathrm{~K}, \log g=2.25,[\mathrm{Fe} / \mathrm{H}]=-0.49$, and a microturbulence of $1.7 \mathrm{~km} \mathrm{~s}^{-1}$ compromise the best-fit averages. We excluded 11 of the 229 spectra that grossly deviated from the bulk, possibly because of problems arising from the continuum level. From the remaining individual values, we took unweighted averages and adopted these as our final atmospheric parameters in Table 3. Their rms deviations are estimated to be the respective internal errors.

The PARSES pipeline returns average $v \sin i$ values between 18 and $24 \mathrm{~km} \mathrm{~s}^{-1}$, depending on the assumed radial-tangential macro- and microturbulence and the wavelength range selected. A much more accurate $v \sin i$ value of $19.4 \pm 0.2 \mathrm{~km} \mathrm{~s}^{-1}$ is possible from our Doppler imaging analysis (see Sect. 4), in good agreement with $19.7 \mathrm{~km} \mathrm{~s}^{-1}$ given by Fekel et al. (1999). The radius of the star derived with the Stefan-Boltzmann law is then

$12.5 \pm 0.5 R_{\odot}$, a typical value for an early-K giant (solar $T_{\text {eff }}$ and $M_{\text {bol }}$ were adopted as $5780 \mathrm{~K}$ and $4 \mathrm{~m} .74$, respectively). The bolometric correction is adopted from Flower (1996). Combination of this radius and the $v \sin i$ leads to an inclination angle of $44^{\circ}$. Simple propagation of the internal errors of the measured and calculated parameters results in an unrealistically small uncertainty for the inclination (just a few degrees). In particular for active giant stars, uncertainties on calculated radius and luminosity are expected to be much larger, hence we estimate $\pm 10^{\circ}$ for the range of the most likely inclination.

Figure 4 compares the position of the star in the $\log T_{\text {eff }}$ vs. $\log L / L_{\odot}$ plane with evolutionary tracks and isochrones from Bertelli et al. (2008). We chose the $Y=0.30$ and $Z=0.008$ tracks, which are closest to but somewhat less metal deficient than our PARSES value (depending on the adopted metallicity of the Sun). The inset in Fig. 4 provides a detailed zoom into the red-giant branch (RGB). The position of HD 208472 suggests a $1.2 M_{\odot}$ mass and an age of $\sim 3.5 \mathrm{Gyr}$. It also indicates that the star is past the RGB bump. Error ranges of $\sim 0.1 M_{\odot}$ in mass and 


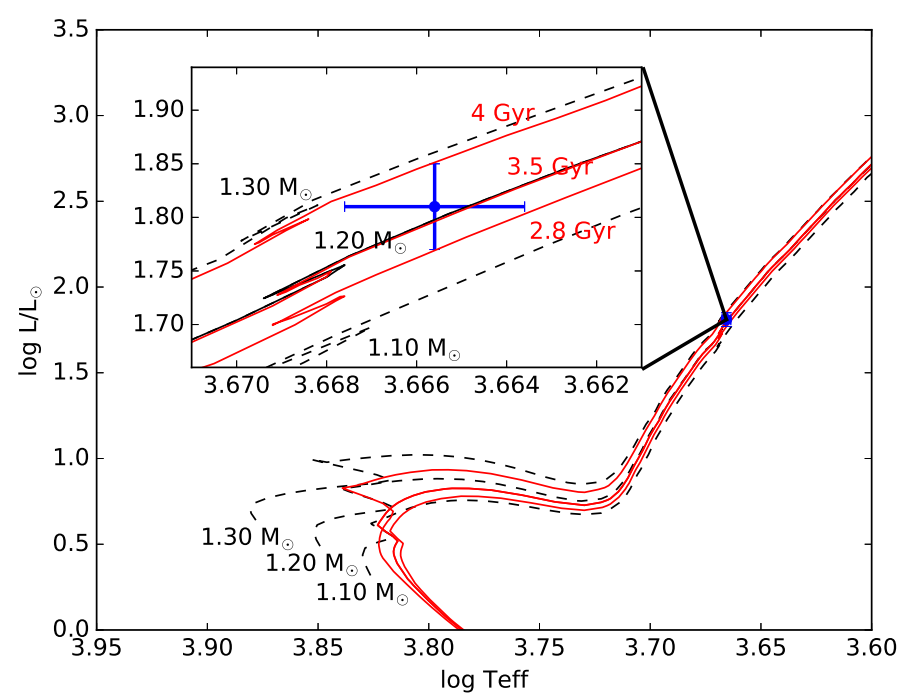

Fig. 4. Position of HD 208472 in the H-R diagram. The tracks (black dashed lines) and isochrones (red continuous lines) are adopted from Bertelli et al. (2008) for $Y=0.30$ and $Z=0.008$.

an age range of 2.8-4.2 Gyr are estimated from the error bars in Fig. 4. A summary of the astrophysical parameters is given in Table 3 .

Because we do not see evidence of the secondary component at optical wavelengths, we expect at least a brightness difference between the components of $\approx 2 \mathrm{~m} .5$. In this case, the secondary component must be fainter than $M_{V} \approx 3 \mathrm{~m}$. 2 . The low-mass function already suggests a low mass for the secondary component. With our best-estimate for the inclination and a $1.2 M_{\odot}$ mass for the primary, we estimate $\sim 0.4 M_{\odot}$ for the secondary mass. This indicates either a white dwarf or a normal M0 dwarf. Unfortunately, there are no ultraviolet observations for HD 208472, and therefore we cannot decide between these two possibilities.

\section{Doppler imaging}

\subsection{Technical setup}

We used our (Zeeman) Doppler-imaging code iMap (Carroll et al. 2007, 2008, 2009, 2012) to reconstruct the surface temperature distribution from time-series STELLA spectra. The code calculates local line profiles by solving the radiative transfer equation under LTE assumption and inverts very many photospheric absorption lines simultaneously. The iteratively regularized Landweber algorithm (Engl et al. 1996; Carroll et al. 2012) is implemented. During calculations, the surface of the star is divided into $5^{\circ} \times 5^{\circ}$ segments; 2592 segments in total. Local line-profile calculations are made for each segment with ATLAS-9 (Castelli \& Kurucz 2004) model atmospheres for the temperature range between $3500 \mathrm{~K}$ and $6000 \mathrm{~K}$ in steps of $250 \mathrm{~K}$ and interpolated to the gravity, metallicity, and microturbulence value of HD 208472. For further details about the code iMap, we refer to Carroll et al. (2012).

\subsection{Line selection and data preparation}

We selected 34 absorption lines that were relatively blend-free for the given temperature and gravity and had a depth larger than $20 \%$ of the continuum. These lines were taken into account simultaneously during the inversion. We applied the multiline
Table 4. Absorption lines used for the mean profile. Atomic data are taken from VALD-3.

\begin{tabular}{llll}
\hline \hline Element & $\lambda(\AA)$ & e.p. $(\mathrm{eV})$ & $\log (g f)$ \\
\hline Fe I & 5049.8197 & 2.279 & -1.335 \\
Fe I & 5307.3607 & 1.608 & -2.987 \\
Fe I & 5322.0407 & 2.279 & -2.803 \\
Cr I & 5348.3140 & 1.004 & -1.210 \\
Fe I & 5367.4659 & 4.415 & +0.443 \\
Fe I & 5383.3685 & 4.313 & +0.645 \\
Fe I & 5393.1672 & 3.241 & -0.715 \\
Fe I & 5415.1989 & 4.387 & +0.642 \\
Fe I & 5497.5160 & 1.011 & -2.849 \\
Fe I & 5569.6180 & 3.417 & -0.486 \\
Fe I & 5572.8423 & 3.397 & -0.275 \\
Fe I & 5576.0888 & 3.430 & -1.000 \\
Ca I & 5581.9650 & 2.523 & -0.555 \\
Fe I & 5862.3564 & 4.549 & -0.127 \\
Ti I & 5866.4513 & 1.067 & -0.790 \\
Fe I & 5956.6940 & 0.859 & -4.605 \\
Fe I & 6021.7889 & 2.198 & -3.925 \\
Fe I & 6024.0575 & 4.549 & -0.120 \\
Ni I & 6108.1159 & 1.676 & -2.600 \\
Ca I & 6122.2170 & 1.886 & -0.316 \\
Fe I & 6173.3343 & 2.223 & -2.880 \\
Fe I & 6200.3125 & 2.609 & -2.437 \\
Ti I & 6261.0988 & 1.430 & -0.530 \\
Fe I & 6265.1323 & 2.176 & -2.550 \\
Fe I & 6358.6967 & 0.859 & -4.468 \\
Fe I & 6393.6004 & 2.433 & -1.432 \\
Fe I & 6421.3499 & 2.279 & -2.027 \\
Fe I & 6430.8450 & 2.176 & -2.006 \\
Ca I & 6439.0750 & 2.526 & +0.390 \\
Ni I & 6643.6304 & 1.676 & -2.220 \\
Fe I & 6663.4411 & 2.424 & -2.479 \\
Ca I & 6717.6810 & 2.709 & -0.524 \\
Fe I & 6750.1515 & 2.424 & -2.621 \\
Ni I & 6767.7721 & 1.826 & -2.140 \\
\hline & & &
\end{tabular}

technique as described in Carroll et al. (2012). Spectral line synthesis was calculated between $\pm 50 \mathrm{~km} \mathrm{~s}^{-1}$ in the velocity domain, however, the velocity range of the line inversion was restricted to $\pm 35 \mathrm{~km} \mathrm{~s}^{-1}$, where the spectral line wings reach the continuum. The lines and their standard parameters from VALD-3 (Kupka et al. 2011) are listed in Table 4.

We divided the spectra into 13 separate data sets, where each set covers one and a half rotational cycles at most. No set has any spectrum from the previous or the following data set in common. This allowed us to trace the spot evolution and differential rotation without a biasing correlation between subsets that would be due to shared data. We summarize the observing log of all 13 data sets in Table 5.

The rotational phase for the spectra is based on our new orbital elements by using the orbital period and a modified epoch as zero point such that one-fourth of the orbital period was subtracted from $T_{0}$ in Table 2 ("spot corrected" column);

$T_{0}(\mathrm{HJD})=2449246.7642+22 \mathrm{~d} .6194 \times E$.

At this modified epoch, the giant component is in inferior conjunction, that is, zero phase is $0^{\circ}$ longitude. We assumed that the star rotates in the counter-clockwise direction and that the 
Table 5. Observing log of Doppler-imaging sets.

\begin{tabular}{lccccc}
\hline \hline Set & Year & $\begin{array}{c}\text { HJD range } \\
(2450000+)\end{array}$ & $N$ & $\begin{array}{c}\text { Max. phase } \\
\text { gap }\end{array}$ & rms \\
\hline 1 & 2009.28 & $4923-4944$ & 15 & 0.13 & 0.0196 \\
2 & 2009.52 & $5009-5033$ & 25 & 0.05 & 0.0021 \\
3 & 2009.59 & $5033-5059$ & 25 & 0.07 & 0.0022 \\
4 & 2009.67 & $5060-5089$ & 25 & 0.09 & 0.0020 \\
5 & 2010.45 & $5348-5370$ & 13 & 0.27 & 0.0019 \\
6 & 2010.53 & $5377-5400$ & 14 & 0.14 & 0.0020 \\
7 & 2010.59 & $5401-5419$ & 10 & 0.20 & 0.0024 \\
8 & 2010.67 & $5429-5451$ & 17 & 0.13 & 0.0019 \\
9 & 2010.73 & $5452-5476$ & 10 & 0.31 & 0.0017 \\
10 & 2010.81 & $5478-5507$ & 12 & 0.47 & 0.0018 \\
11 & 2011.36 & $5685-5699$ & 7 & 0.38 & 0.0018 \\
12 & 2011.47 & $5722-5742$ & 17 & 0.13 & 0.0020 \\
13 & 2011.53 & $5743-5763$ & 12 & 0.26 & 0.0020 \\
\hline
\end{tabular}

Notes. The year is given for the mid-times of the related sets. $N$ is the number spectra per set, and max-phase gap is the largest rotational phase gap between two successive observation. The error value of each inverted set is given in the rms column.

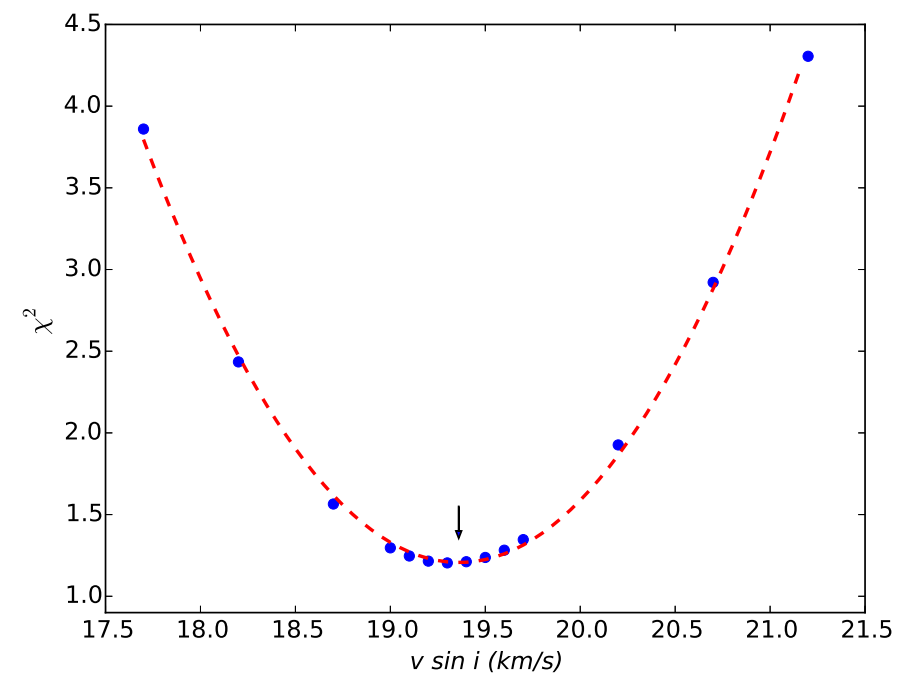

Fig. 5. Quality-of-fit $\left(\chi^{2}\right)$ for a series of trial $v \sin i$ values. The red dashed curve is a quadratic fit to the data points. The arrow indicates the position of the best-fit $v \sin i$ of $19.4 \mathrm{~km} \mathrm{~s}^{-1}$.

longitude increases in the same direction so that phase 0.25 ( $\phi=0.25$ ) corresponds to $270^{\circ}$ longitude on the stellar surface, for instance.

\section{3. $v \sin i$ validation}

Before calculating surface maps, we searched for the $v \sin i$ value that provided the best representation for the rotationally broadened line profiles. For this purpose, we chose the third data set from the 2009 observing season, which is one of the best sets with very good sampling and high $\mathrm{S} / \mathrm{N}$. We reconstructed surface images for this set by iterating $v \sin i$ values between 17.7 and $21.2 \mathrm{~km} \mathrm{~s}^{-1}$ while keeping the remaining parameters fixed. A parabolic fit to the distribution of $\chi^{2}$ in Fig. 5 indicates a bestmatch $v \sin i$ of $19.4 \pm 0.2 \mathrm{~km} \mathrm{~s}^{-1}$. The error comes from the width of the parabola, $\pm 0.2 \mathrm{~km} \mathrm{~s}^{-1}$ steps in the sampling, where the deviation is $1 \sigma$. We adopted this value as our best estimate and kept it fixed for the remaining imaging.

\section{Results}

\subsection{Surface maps}

Figures 6 to 8 present our Doppler images for 2009 to 2011. We plot observed and inverted line profiles of each set in Fig. A.1. None of the images shows a dominant polar spot, in agreement with earlier maps by Weber (2004). Spots on HD 208472 are generally not very large and on average seem cooler than the undisturbed photosphere by just $\sim 500 \mathrm{~K}$. We did not recover any bright features warmer than the photosphere.

Spots typically appear at latitudes higher than $\approx 30^{\circ}$, except for a few cases where an extension of a larger spot may bridge that latitude (e.g., in 2010, sets 5 and 9). The longitudinal concentration, on the other hand, had changed abruptly at one point. While in 2009, spots concentrated around zero phase $(\phi=0.00$; facing the observer at inferior conjunction), in the following season in 2010 the main concentration occurred near $\phi=0.50$ and remained so into 2011 until the end of our data set. We focus on this change in Sect. 5.2, but first we describe the results from the individual observing seasons.

2009 observing season. We have complete phase coverage for each image reconstructed from 2009. In the first image (set 1), we reconstruct three individual spots with latitudes between $45^{\circ}$ and $75^{\circ}$ and separated by $\sim 0.40$ in phase. The second image (set 2 ) shows the larger of the three spots at $\sim 60^{\circ}$ latitude and at $\phi=0.05$. Two of the spots seem to be marginally connected with each other, and this region appears to evolve systematically. Because there are almost four rotations between sets 1 and 2, some spot evolution is even expected. As time progresses from set 2 to set 4 (i.e., until the last image in 2009), the main spot at $\phi=0.05$ still exists but appears to have drifted toward decreasing phases with respect to the epoch and orbital period in Eq. (1). Spot migration like this is also typical for the shifts expected from differential rotation. One of the two originally connected spots had continuously drifted toward the larger spot. It had completely disappeared by the end of the season.

2010 observing season. In 2010 the phase coverage is not as good as in 2009, but still quite good for reconstructing reliable surface maps. The time gap between successive maps is typically a few days, except between sets 7 and 8, where it is ten days. Nevertheless, spot evolution can be traced safely through six successive stellar rotations. In the beginning of 2010 (set 5), we see the main spot at $\phi=0.83$ and two weaker spots at $\phi=0.50$ and $\phi=0.18$ at lower latitudes than the main spot. As time progresses, the spot at $\phi=0.18$ fades, while the spot at $\phi=0.50$ cools and becomes the most dominant feature. The spot at $\phi=0.83$ still survives but warms up and eventually approaches the main spot at $\phi=0.50$. We also observe that the two spots are connected to each other with a pale structure around $\sim 75^{\circ}$ latitude that is slightly cooler than the photosphere. All spots appear paler in set 7 , while these spots appeared darker in the previous and in the following sets. This is most likely related to the data quality of set 7 , where the average $\mathrm{S} / \mathrm{N}$ is just around 120 , while it is usually between 170-200. Moreover, the visible surface from sets 9 and 10 at $\phi=0.00$ appears to be almost unspotted except for the high-latitude features close to the rim of the visible surface. We emphasize that sets 9 and 10 have considerable phase gaps (listed in Table 5). We have no observation between $\phi=0.75$ and $\phi=0.25$ in set 10 and $\phi=0.70$ and $\phi=0.00$ in set 9 . The consequence is that whatever weak structure may have existed at these longitudes, it cannot be reconstructed. The sudden disappearance of spots within these phases is related to these phase gaps and not to spot decay, for example. 


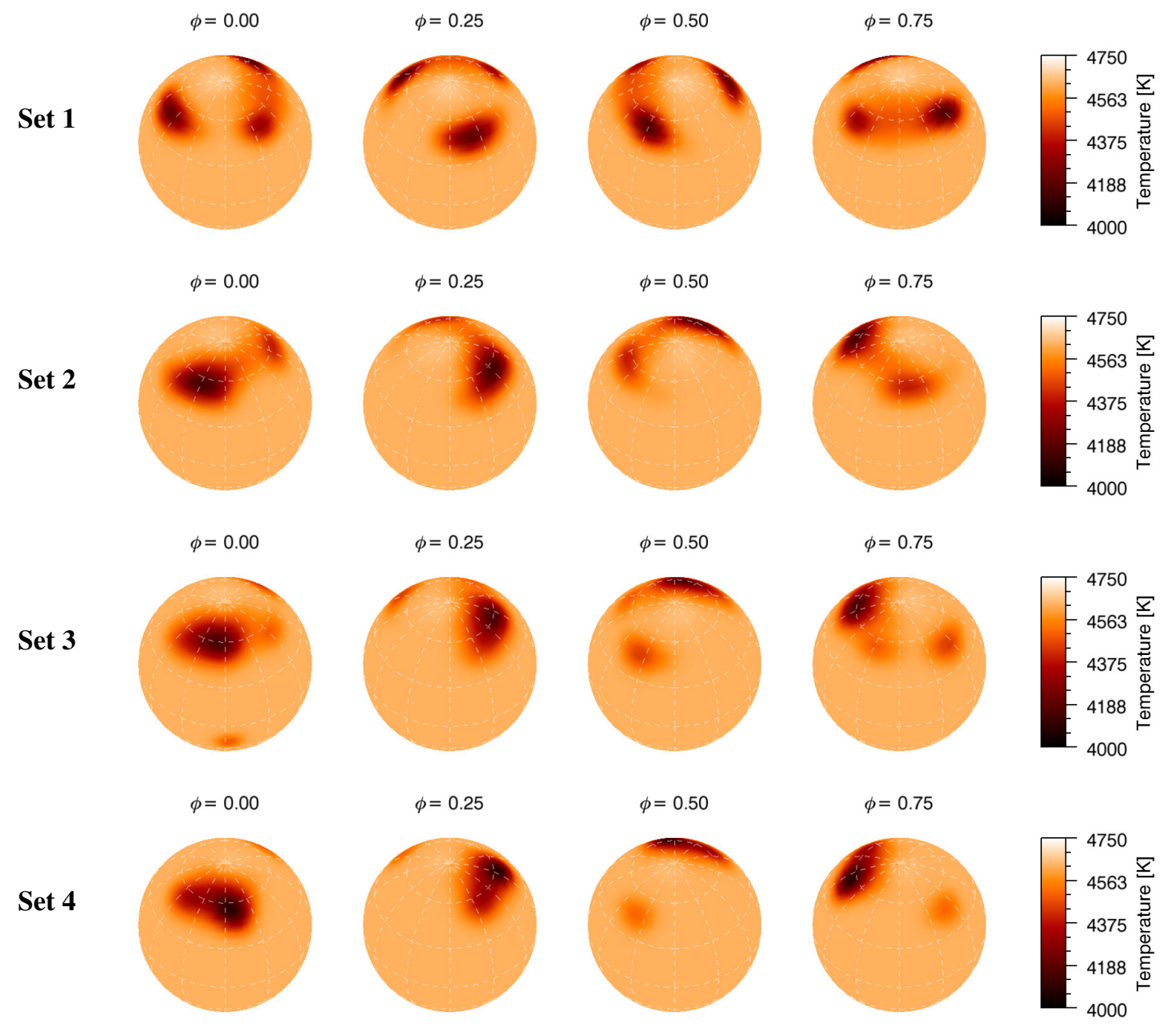

Fig. 6. Doppler images of HD 208472 for the 2009 observing season.

2011 observing season. We have three maps for 2011 covering four consecutive stellar rotations. There is a time gap of one rotation between the end of set 11 and the beginning of set 12 . We note that set 11 comprises only seven spectra with a maximum phase gap between two successive observations of 0.38 between $\phi=0.28$ and $\phi=0.66$. Care must be taken to avoid overinterpreting spots at these phases. Set 11 shows one cool, dominant spot at $\phi=0.40$ and two warmer spots at $\phi=0.70$ and $\phi=0.00$. After two rotations (i.e., in set 12), the main spot appears to have broken into two smaller and warmer spots, while the spot at $\phi=0.70$ became the most dominant spot. However, this evolution scenario is hampered by the phase gaps in set 11 and is not conclusive. The spot at $\phi=0.00$ seems to have moved toward decreased phases by the time of set 12 and then has vanished in set 13. At the same time, a small appendage of the originally cooler spot has migrated to $\phi=0.10$.

\subsection{Active longitudes}

In Paper I we showed that an activity cycle repeats almost regularly in time. A preliminary 6.28-yr activity cycle was reconstructed from long-term photometry. It predicted a new activity cycle to begin around 2009/2010. In the previous section, we noted that the main spot activity was located around $\phi=0.00$ in 2009, while it was around $\phi=0.50$ in 2010 and 2011. There were also almost no spots at $\phi=0.50$ in the 2009 maps, while the main spot concentration was observed at exactly that phase in 2010 and 2011. This means that we apparently witnessed a flip-flop event. The new longitudes of the main activity zone appear to correspond to either the sub-stellar point (i.e., $\phi=0.00$ ) or its antipode $(\phi=0.50)$. We interpret this as evidence for two persistent active longitudes separated by $180^{\circ}$ on the surface of HD 208472, which might be related with the binary nature of the star.

We also applied the same two-spot model technique as in Paper I to our recent $V$-band photometry from 2010-2015. In this way, we obtained the longitudinal distribution of spots, but over a longer period in time than the Doppler imaging. For details of the technique we refer to Paper I and references therein. The results are shown in Fig. 9a, which is the updated version of the Fig. 10 in Paper I. The spot positions show the continuing systematic drifts and verify the beginning of the new activity cycle around 2009/2010. Furthermore, the most recent photometric data show some evidence for yet another phase jump in 2015, in agreement with the prediction from the $\sim 6 \mathrm{yr}$ cycle length. The light curves in the orbital phase frame are shown in Fig. 9 b. The active longitudes clearly show the alternating locations of minima, but the figure also emphasizes the existence of the ever increasing brightness of the system (see Fig. 1).

\subsection{Differential rotation}

We traced the position of spots by cross-correlating successive Doppler maps in longitudinal direction and examining the 


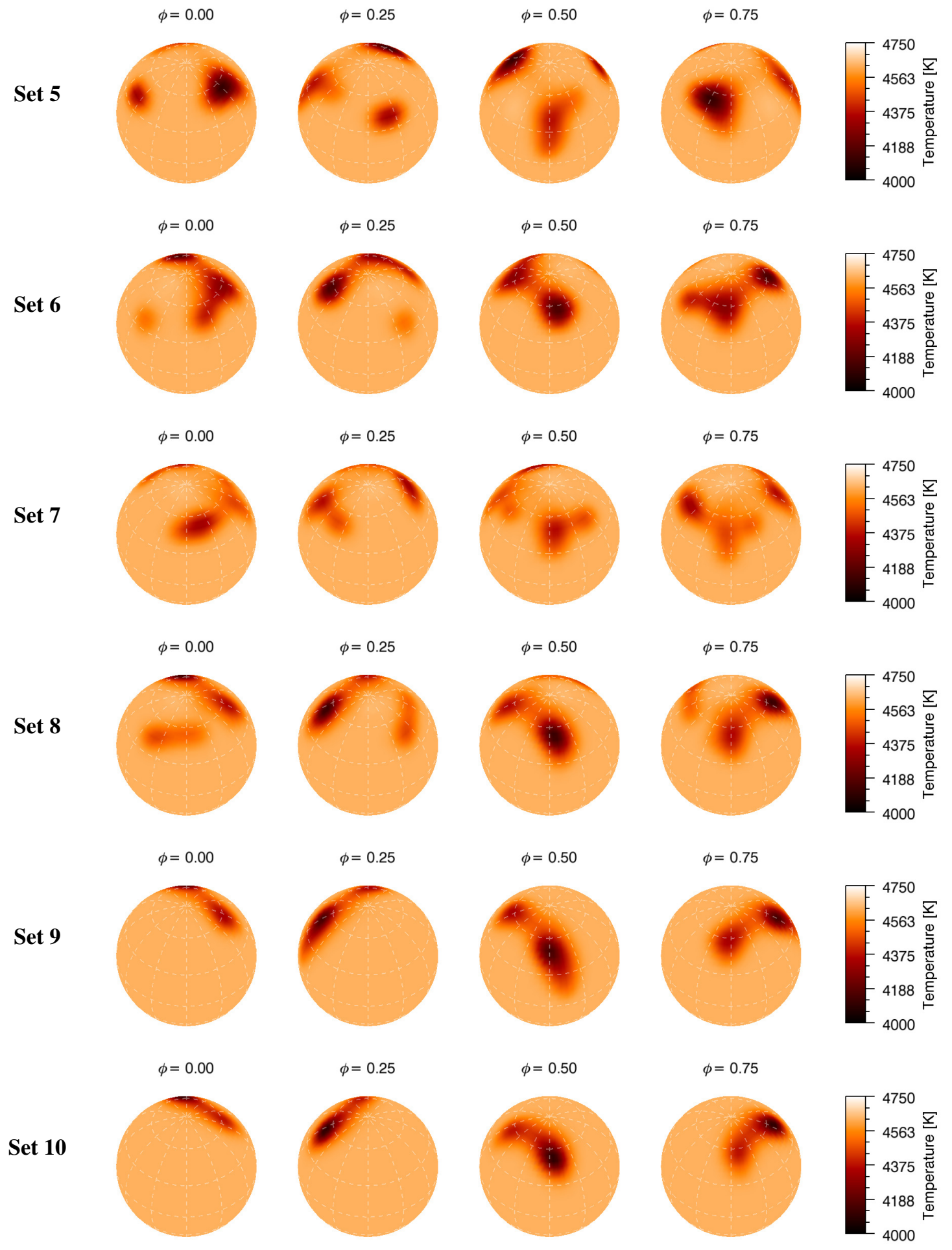

Fig. 7. Doppler images of HD 208472 for the 2010 observing season.

amount of longitudinal shift in a given latitude range where spots occur (e.g., Kővári et al. 2015; Künstler et al. 2015). This is basically equivalent to tracking the motion of sunspots as applied to solar-disk images (e.g., Howard 1984; Brajša et al. 2002).

In our present application, we are able to produce three crosscorrelation-function (ccf) maps for the observing season 2009, five ccf maps for 2010, and two ccf maps for 2011. Altogether, we have ten ccf maps at our disposal. Because the Doppler images are based on a $5^{\circ} \times 5^{\circ}$ equal-degree segmentation of the surface, we also calculated the cross correlation for latitudinal bins of $5^{\circ}$ width. A Gaussian profile was used for fitting and identifying the CCF peaks. We present the grand average ccf map in Fig. 10a, which was obtained by averaging all ten ccf maps from three consecutive years. It shows that the available spot-displacement information extends from the visible pole down to $\approx 10^{\circ}$ latitude. Thus, HD 208472 provides a wide latitude range with spots and indeed allows for a good estimation of its differential rotation. In Fig. 10b we plot the positions of 


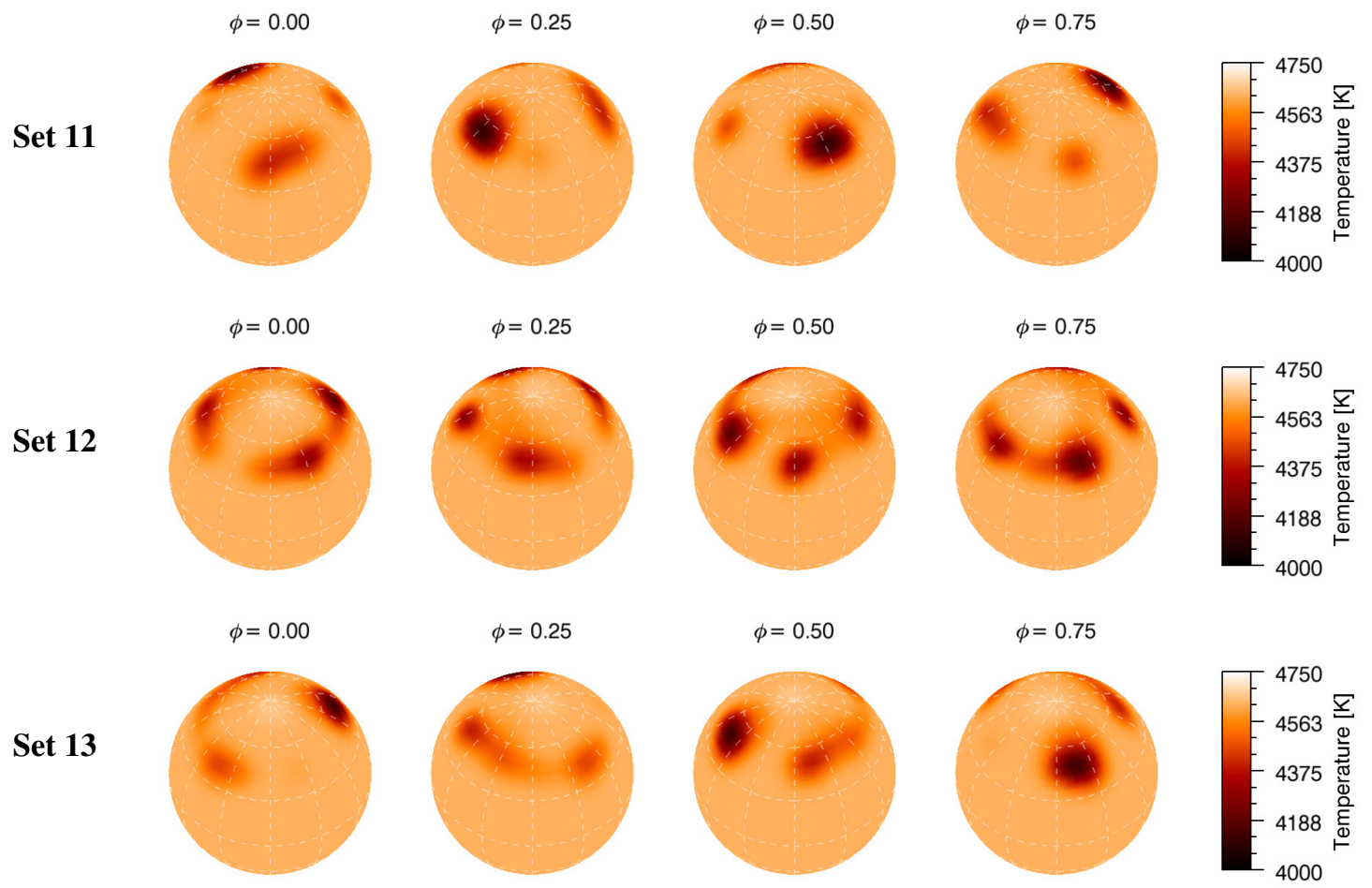

Fig. 8. Doppler images of HD 208472 for the 2011 observing season.

a)

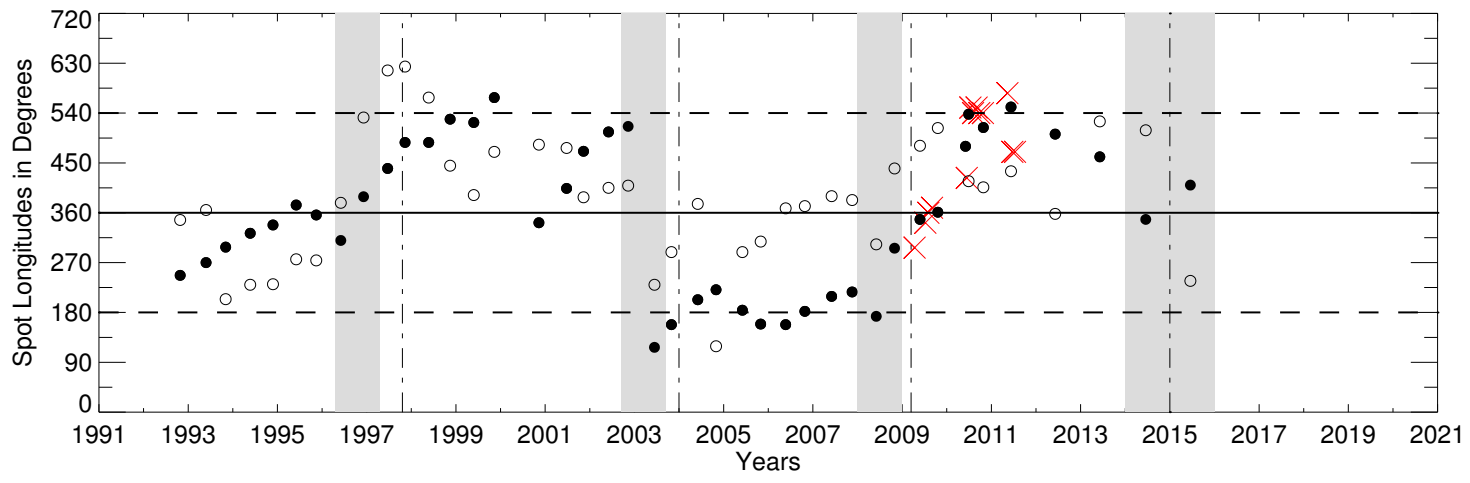

b)

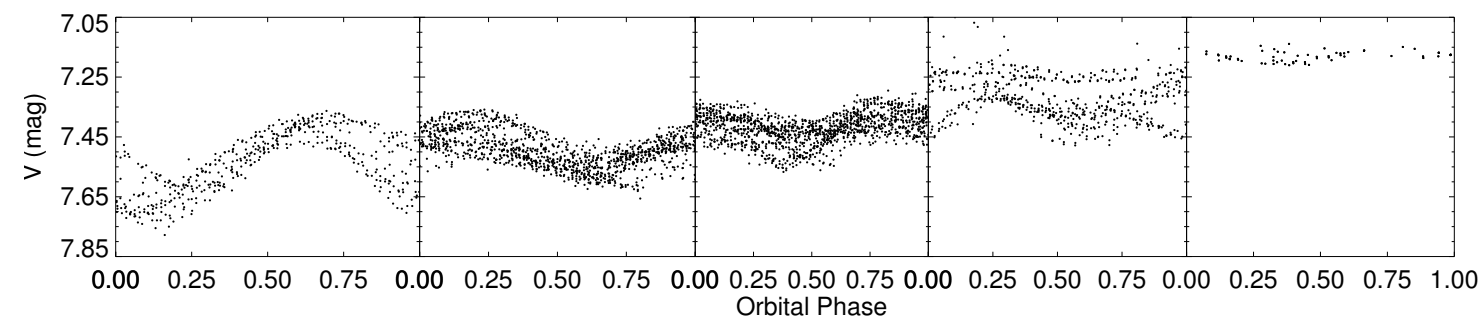

Fig. 9. a) Longitudinal distribution of spots on HD 208472 from photometric two-spot models. The shaded regions are the time ranges where spots change their preferential positions (hemispheres) on the surface of the star. The continuous line represents the longitudes of the apsidal line of the binary system, while the two dashed lines restrict the stellar surface. The vertical dot-dashed lines correspond to times when changes occurred in light-curve amplitude and photometric period. We overplot the longitudes of the main spot of the Doppler images with cross symbols in red. b) Accumulated $V$ brightness phased with the ephemeris in Eq. (1), and separated into the corresponding time intervals in panel a).

the ccf peaks along with their estimated errors defined as the full-width at half maximum of the respective Gaussian fits. We then fit a solar-like differential rotation law to the global ccf pattern of the star. Considering the angular velocities at a latitude $b$ and at the equator $\left(\Omega(b)\right.$ and $\Omega_{\mathrm{eq}}$, respectively), and the difference between the angular velocities at the pole and the equator
$\left(\Delta \Omega=\Omega_{\mathrm{eq}}-\Omega_{\mathrm{pole}}\right)$, we adopt a typical differential rotation law as

$\Omega(b)=\Omega_{\mathrm{eq}}-\Delta \Omega \sin ^{2}(b)$,

where the surface shear parameter, $\alpha$, is defined by $\alpha=\Delta \Omega / \Omega_{\mathrm{eq}}$. Using Eq. (2) to fit the differential rotation pattern in Fig. 10b, 


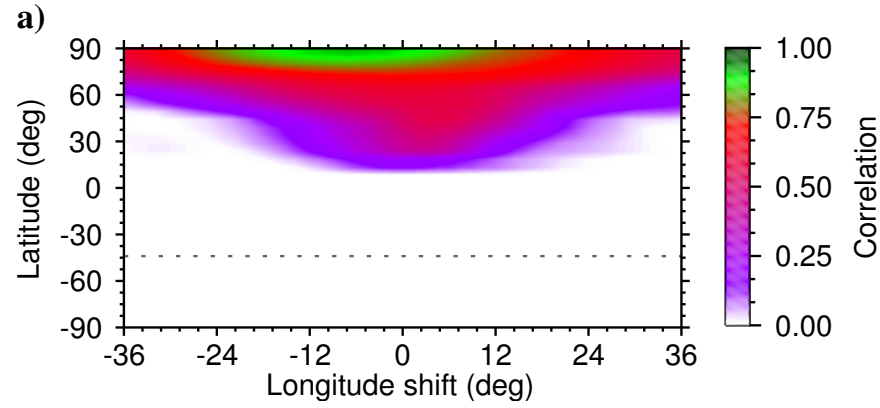

b)

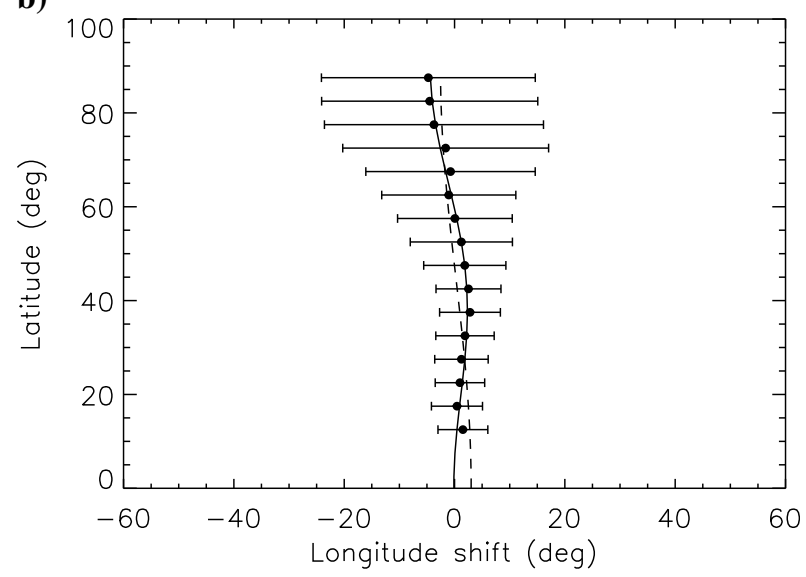

Fig. 10. a) Average ccf map from ten seasonal ccf maps. b) Average differential rotation pattern. The dashed line denotes the best fit using the single-term law in Eq. (2), while the solid line shows the best fit from the double-term law in Eq. (3).

we find $\alpha=+0.015 \pm 0.003$ and $\Omega_{\mathrm{eq}}=0.2801 \pm 0.0004 \mathrm{rad} / \mathrm{day}$, which corresponds to an equatorial period $P_{\text {eq }}=22.431 \pm$ $0.031 \mathrm{~d}$.

A better representation of the differential rotation pattern can be achieved by adding another term to the differential rotation law, which is commonly adopted for the Sun,

$\Omega(b)=\Omega_{\mathrm{eq}}+\Omega_{1} \sin ^{2}(b)+\Omega_{2} \sin ^{4}(b)$,

where $\Omega_{\text {pole }}$ is given by the sum of $\Omega_{\mathrm{eq}}, \Omega_{1}$, and $\Omega_{2}$. In this case, we find a shear parameter $\alpha$ of $+0.012 \pm 0.008, \Omega_{\mathrm{eq}}=$ $0.2777 \pm 0.0006 \mathrm{rad} / \mathrm{day}$ and $P_{\text {eq }}=22.628 \pm 0.048 \mathrm{~d}$, where $\Omega_{1}=$ $0.0101 \pm 0.0016 \mathrm{rad} /$ day and $\Omega_{2}=-0.0134 \pm 0.0015 \mathrm{rad} /$ day.

Both obtained values of $P_{\text {eq }}$ differ by more than their individual errors. This discrepancy is caused by the lack of data points below a latitude of $\pm 10^{\circ} \mathrm{deg}$. The two $\alpha$ values agree to within their errors and suggest weak but solar-type differential rotation with a surface shear about one order of magnitude weaker than for the Sun. Such weak differential rotation is a common result for RS CVn binary stars with late-type cool giants, such as for XX Tri (Künstler et al. 2015) or $\zeta$ And (Kővári et al. 2012), for which both studies were based on time-series Doppler imaging.

\section{Summary and conclusions}

Three years of high-resolution time-series STELLA spectra enabled us to not only refine precise spectroscopic orbital elements and atmospheric parameters of HD 208472, but reconstruct 13 separate surface images from three observing seasons through multiple-line inversions. Surface reconstructions were made by tracing the distortions in the absorption line profiles with time, which is the main principle of our time-series approach of Doppler imaging. The line-profile distortions are also the cause of systematic shifts in radial velocities, known as spot jitter in case of chromospherically active stars. After proper removal, it decreased the RV residuals for our new spectroscopic orbital solution by a factor of four from $380 \mathrm{~m} \mathrm{~s}^{-1}$ to $88 \mathrm{~m} \mathrm{~s}^{-1}$. Fourier analysis of the RV residuals indicated that the dominant periods are always very close to the orbital period and its harmonics, which is the typical signature of spot jitter.

HD 208472 exhibits active longitudes on adjacent hemispheres and occasional flip-flops between them that overlap the continuous drift in longitude with respect to the orbital reference frame. Similar active longitudes and flip-flops were observed in several RS CVn binaries (Jetsu 1996; Berdyugina \& Tuominen 1998; Künstler et al. 2015), FK Com-type stars (Jetsu et al. 1991, 1999), and young solar-like stars (Berdyugina \& Järvinen 2005). There is some evidence that flip-flops occur more or less regularly with timescales of a few years up to a decade, sometimes referred to as flip-flop cycle (see the references above), but the evidence remains inconclusive. To detect these cycle lengths, we still need long-term photometry with phase-resolved sampling or, even better, modern time-series Doppler imaging.

A 6.28 yr flip-flop cycle was discovered for HD 208472 in our Paper I, where we predicted the begin of a new cycle for 2009/2010. Our Doppler imagery in the present paper caught this event. We witnessed the change of the main activity zone from $\phi=0.00$ to $\phi=0.50$ between the 2009 and 2010 observing seasons over a time range of approximately one year. Thus, the location of the main activity zone corresponds to the substellar point and its antipode, as has been suggested by Oláh (2006). Theoretically, coexistence of axisymmetric and nonaxisymmetric dynamo modes may produce active longitudes and also give rise to the flip-flop phenomenon (Fluri \& Berdyugina 2004; Moss 2005; Elstner \& Korhonen 2005). However, why these are aligned and influenced by the secondary star and why the brightness continues to increase over several activity cycle is yet to be understood.

From cross-correlating successive Doppler maps, we found a surface shear of $\alpha=+0.015 \pm 0.003$ for a $\sin ^{2}$-latitude differential rotation law and $\alpha=+0.012 \pm 0.008$ for a $\sin ^{2}+\sin ^{4}$ latitude differential rotation law. This differential rotation is in solar-like direction, meaning that poles rotate slower than the equator, but $\approx 15$ times weaker than on the Sun. The average value is close to the $\alpha$ value of XX Tri (Künstler et al. 2015), which is a similar RS CVn system in terms of physical properties and spectroscopic orbit, but maybe about twice as old as HD 208472. Comparing other stars whose $\alpha$ values were determined from Doppler imaging, our target star belongs to a group of weak solar-type differential rotators

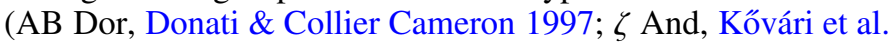
2012; IL Hya, Kővári et al. 2014). On the other hand, anti-solar differential rotation was found (or at least claimed) in some other active stars, such as $\sigma$ Gem (Kővári et al. 2015), UZ Lib (Vida et al. 2007), HU Vir (Strassmeier 1994; Harutyunyan et al. 2016), and HD 31993 (Strassmeier et al. 2003). Tidal force in a binary system is considered as a possible explanation for the existence of two different directions of differential rotation, depending on the orbital and physical properties of the related system. Holzwarth \& Schüssler (2002) showed that the tidal force in an RS CVn binary may lead to preferred longitudes. It may also affect the angular momentum distribution in the convective envelope of the giant component and alter its differential rotation pattern (Kóvári et al. 2012). Another effect of the tidal force on differential rotation might be the suppression of the strength of 
the differential rotation through tidal locking (Collier Cameron 2007). The RS CVn binaries mentioned above have $\alpha$ values of just a few of per cent (solar or anti-solar). In comparison, the single K2 giant HD 31993 (Strassmeier et al. 2003), where no tidal interaction is present, has a dramatically higher shear value of $\alpha=-0.125$. Another comparison can be made with the single K1 giant DP CVn (Kővári et al. 2013), which has a surface shear value of $\alpha=-0.035$, which is a stronger surface shear in magnitude than HD 208472. This shows that fast-rotating single giants with $\alpha$ values even comparable to the solar value have been reported, while no such RS CVn stars have been found. This supports the picture that differential rotation is suppressed by tidal forces as suggested by Scharlemann (1982), for example.

Acknowledgements. O.Ö. thanks the Scientific and Technological Research Council of Turkey (TÜBİTAK) for funding this study under "2219 post-doc research scholarship program". O.Ö. appreciates friendly and warm hospitality of AIP Stellar Activity Group during his post-doc research at AIP. K.G.S. thanks the State of Brandenburg and the German BMBF for the continuous funding of the STELLA facility in Tenerife and the APTs in southern Arizona.

\section{References}

Allende Prieto, C. 2004, Astron. Nachr., 325, 604

Allende-Prieto, C., Beers, T. C., Wilhelm, R., et al. 2006, ApJ, 636, 804

Berdyugina, S. V., \& Järvinen, S. P. 2005, Astron. Nachr., 326, 283

Berdyugina, S. V., \& Tuominen, I. 1998, A\&A, 336, L25

Bertelli, G., Girardi, L., Marigo, P., \& Nasi, E. 2008, A\&A, 484, 815

Brajša, R., Wöhl, H., Vršnak, B., et al. 2002, Sol. Phys., 206, 229

Carroll, T. A., Kopf, M., Ilyin, I., \& Strassmeier, K. G. 2007, Astron. Nachr., 328,1043

Carroll, T. A., Kopf, M., \& Strassmeier, K. G. 2008, A\&A, 488, 781

Carroll, T. A., Kopf, M., Strassmeier, K. G., Ilyin, I., \& Tuominen, I. 2009, in Cosmic Magnetic Fields: From Planets, to Stars and Galaxies, IAU Symp., 259,437

Carroll, T. A., Strassmeier, K. G., Rice, J. B., \& Künstler, A. 2012, A\&A, 548, A95

Castelli, F., \& Kurucz, R. L. 2004, ArXiv e-prints [arXiv: astro-ph/0405087]

Collier Cameron, A. 2007, Astron. Nachr., 328, 1030

Donati, J.-F., \& Collier Cameron, A. 1997, MNRAS, 291, 1

Donati, J.-F., Collier Cameron, A., \& Petit, P. 2003, MNRAS, 345, 1187

Dunstone, N. J., Hussain, G. A. J., Collier Cameron, A., et al. 2008, MNRAS, 387,1525

Elstner, D., \& Korhonen, H. 2005, Astron. Nachr., 326, 278

Engl, H. W., Hanke, \& M. Neubauer, A. 1996, Regularization of Inverse problems, Mathematics and Its application (Dordrecht, The Netherlands: Kluwer Academic Publishers)

Erdem, A., Budding, E., Soydugan, E., et al. 2009, New Astron., 14, 545

Fekel, F. C., Strassmeier, K. G., Weber, M., \& Washuettl, A. 1999, A\&AS, 137, 369

Flower, P. J. 1996, ApJ, 469, 355

Fluri, D. M., \& Berdyugina, S. V. 2004, Sol. Phys., 224, 153
Gray, D. F. 2005, The observation and analysis of stellar photospheres, 3rd edn. (Cambridge Univ. Press)

Gustafsson, B., Edvardsson, B., Eriksson, K., et al. 2008, A\&A, 486, 951

Harutyunyan, G., Strassmeier, K. G., Künstler, A., Carroll, T., \& Weber, M. 2016, A\&A, 592, A117

Henry, G. W., Fekel, F. C., \& Hall, D. S. 1995, AJ, 110, 2926

Holzwarth, V., \& Schüssler, M. 2002, Astron. Nachr., 323, 399

Holzwarth, V., \& Schüssler, M. 2003a, A\&A, 405, 291

Holzwarth, V., \& Schüssler, M. 2003b, A\&A, 405, 303

Howard, R. 1984, ARA\&A, 22, 131

Jetsu, L. 1996, A\&A, 314, 153

Jetsu, L., Pelt, J., Tuominen, I., \& Nations, H. 1991, in The Sun and Cool Stars: activity, magnetism, dynamos, eds. I. Tuominen, D. Moss, \& G. Rüdiger, (Berlin: Springer), Proc. IAU Colloq., 130, 381

Jetsu, L., Pelt, J., \& Tuominen, I. 1999, A\&A, 351, 212

Jovanovic, M., Weber, M., \& Allende Prieto, C. 2013, Publ. Obs. Astron. Belgrade, 92, 169

Kitchatinov, L. L., \& Rüdiger, G. 2004, Astron. Nachr., 325, 496

Koen, C., \& Eyer, L. 2002, MNRAS, 331, 45

Korhonen, H., \& Elstner, D. 2011, A\&A, 532, A106

Kővári, Z., Korhonen, H., Kriskovics, L., et al. 2012, A\&A, 539, A50

Kővári, Z., Korhonen, H., Strassmeier, K. G., et al. 2013, A\&A, 551, A2

Kővári, Z., Kriskovics, L., Oláh, K., et al. 2014, in Magnetic Fields throughout Stellar Evolution, Proc. IAU Symp. (Cambridge University Press), 302, 379

Kővári, Z., Kriskovics, L., Künstler, A., et al. 2015, A\&A, 573, A98

Kővari, Z., Künstler, A., Strassmeier, K. G., et al. 2016, A\&A, submitted

Kupka, F., Dubernet, M.-L., et al. 2011, Balt. Astron., 20, 503

Künstler, A., Carroll, T. A., \& Strassmeier, K. G. 2015, A\&A, 578, A101

Lenz, P., \& Breger, M. 2004 in The A-Star Puzzle, eds. J. Zverko, J. Žižnovsky̌, S. J. Adelman \& W. W. Weiss, IAU Symp., 224, 786

Moss, D. 2005, A\&A, 432, 249

Oláh, K. 2006, Ap\&SS, 304, 145

Oláh, K., Kővari, Z., Bartus, J., et al. 1997, A\&A, 321, 811

Özdarcan, O., Evren, S., Strassmeier, K. G., Granzer, T., \& Henry, G. W. 2010, Astron. Nachr., 331, 794 (Paper I)

Petit, P., Donati, J.-F., Wade, G. A., et al. 2004, MNRAS, 348, 1175

Roettenbacher, R. M., Monnier, J. D., Henry, G. W., et al. 2015a, ApJ, 807, 23

Roettenbacher, R. M., Monnier, J. D., Fekel, F. C., et al. 2015b, ApJ, 809, 159

Scharlemann, E. T. 1982, ApJ, 253, 298

Strassmeier, K. G. 1994, A\&A, 281, 395

Strassmeier, K. G. 2009, A\&ARv, 17, 251

Strassmeier, K. G., Boyd, L. J., Epand, D. H., \& Granzer, T. 1997, PASP, 109, 697

Strassmeier, K. G., Serkowitsch, E., \& Granzer, T. 1999, A\&AS, 140, 29

Strassmeier, K. G., Kratzwald, L., \& Weber, M. 2003, A\&A, 408, 1103

Strassmeier, K. G., Weber, M., Granzer, T., Järvinen, S. 2012, Astron. Nachr., 333,663

van Leeuwen, F. 2007, A\&A, 474, 653

Vida, K., Kovári, Z., Švanda, M., et al. 2007, Astron. Nachr., 328, 1078

Vogt, S. S., Hatzes, A. P., Misch, A. A., Kürster, M. 1999, ApJS, 121, 547

Weber, M. 2004, Ph.D. Thesis, University of Potsdam, Germany

Weber, M., Strassmeier, K. G., \& Washuettl, A. 2001, in Proc. of the JENAM, Munich, Astron. Ges. Abstract Series, 18, 89

Weber, M., Strassmeier, K. G., \& Washuettl, A. 2005, Astron. Nachr., 326, 287

Weber, M., Granzer, T., Strassmeier, K. G., \& Woche, M. 2008, in SPIE Conf. Ser., 7019, 70190L 


\section{Appendix A: Line profiles of Doppler images}

Figure A.1 shows observed and inverted line profiles of each Doppler image given in Figs. 6 to 8 .
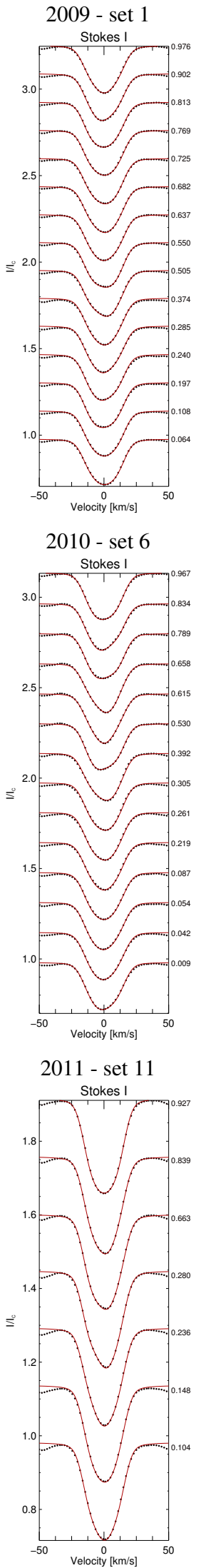

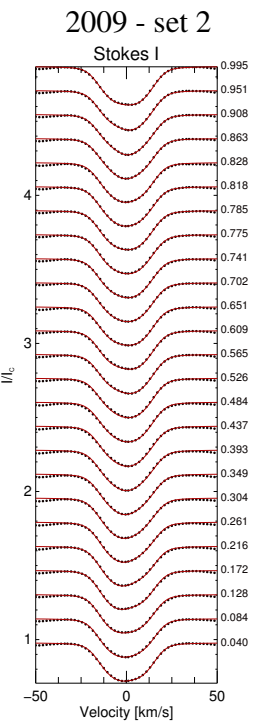

2010 - set 7



2011 - set 12

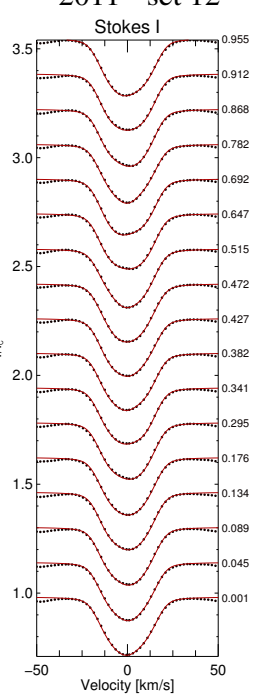



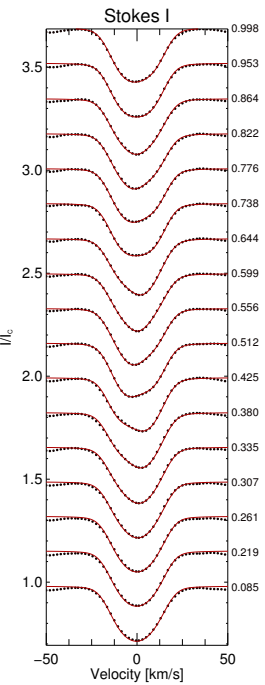

2011 - set 13

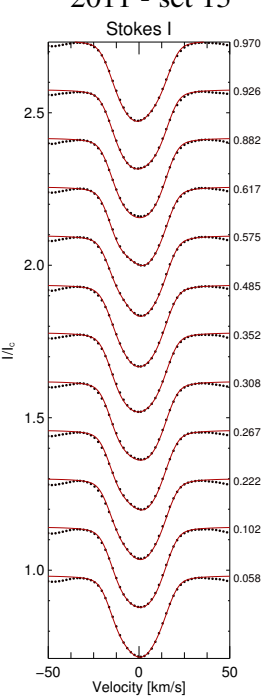

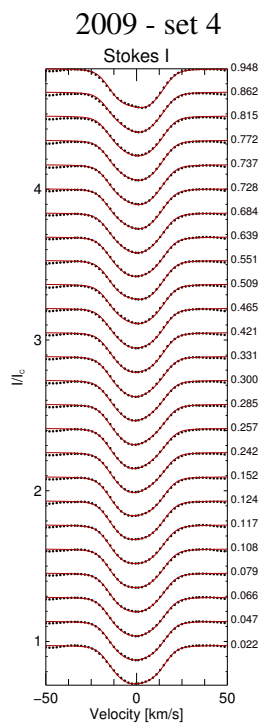

2010 - set 9

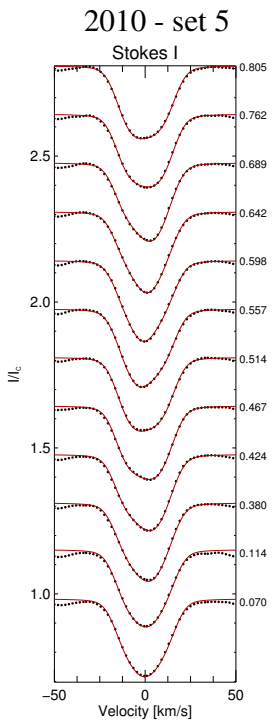

2010 - set 10
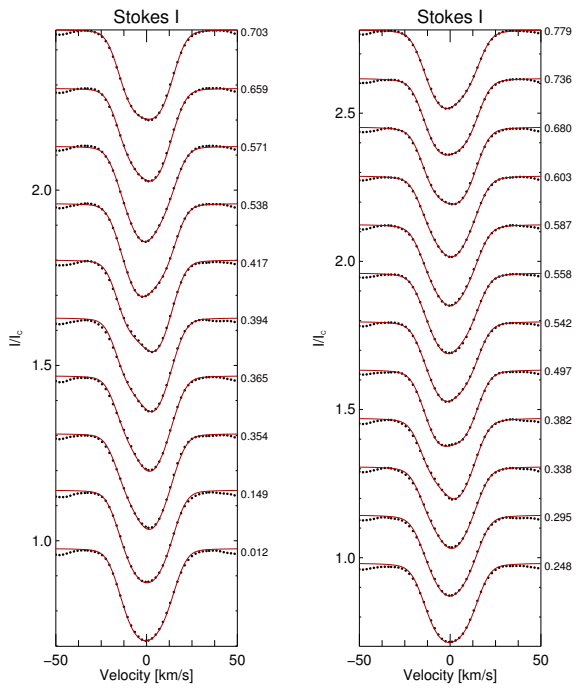

Fig. A.1. Observed (dots) and inverted (continuous curve) line profiles of 13 sets. For a given set, the corresponding orbital phase of each line profile is shown at the right side of the related panel. Observing season and set numbers are shown at the top of the panels. 